\title{
Locating shifts in the unconditional variance of time-dependent processes using multiscale wavelet periodogram
}

\begin{abstract}
In this paper, we offer a proxy volatility measure based on maximum overlap discrete wavelet transform (MODWT) called multiscale wavelet periodogram (MSWP). We show that the multiscale wavelet periodogram (MSWP) is well equipped to detect multiple changes in the unconditional variance of a time-varying volatile process. Our simulation results demonstrate that use of MSWP as an input instead of the squared variable can overcome the size distortion problem associated with the CUSUM-type test for the detection of shifts in the unconditional variance. The power of the test based on wavelet periodograms is in fact comparable to that of the test based on squared variable. Our new approach is found successful for analyzing real data.
\end{abstract}

Keyword: Multiscale wavelet periodogram; Volatility; Variance shift; CUSUM-type test 